\title{
The impact of coronary perforation in percutaneous interventions involving the left main stem coronary artery in the United Kingdom 2007-2014: Insights from the British Cardiovascular Intervention Society database
}

\author{
Hussain I. Hussain MD ${ }^{1} \quad$ ～Majd B. Protty PhD ${ }^{1,2}$ (c) | Sean Gallagher $\mathrm{MD}^{1} \quad$ | \\ Sara Al-Raisi MD ${ }^{1}$ | Omar Aldalati MD ${ }^{1}$ | Vasim Farooq MD $^{1}$ | \\ Andrew S. P. Sharp PhD ${ }^{1,3} \quad$ Richard Anderson MD ${ }^{1}$ (1) | Tim Kinnaird MD MD $^{1,4}$
}

\footnotetext{
${ }^{1}$ Department of Cardiology, University Hospital of Wales, Cardiff, UK

${ }^{2}$ Systems Immunity University Research Institute, Cardiff University, Cardiff, UK

${ }^{3}$ University of Exeter, Exeter, Devon, UK

${ }^{4}$ Keele Cardiovascular Research Group, Institute of Applied Clinical Sciences, University of Keele, Stoke-on-Trent, UK

Correspondence

Tim Kinnaird, Consultant Interventional Cardiologist, Department of Cardiology, University Hospital of Wales, Cardiff CF14 4XW, UK.

Email: tim.kinnaird2@wales.nhs.uk
}

\begin{abstract}
Background: Percutaneous coronary intervention $(\mathrm{PCI})$ is increasingly utilized for treatment of coronary disease involving the unprotected left main stem (ULMS). However, no studies to date have examined the outcomes of such interventions when complicated by coronary perforation (CP).

Methods: Using the British Cardiovascular Intervention society (BCIS) database, data were analyzed on all ULMS-PCI procedures complicated by CP in England and Wales between 2007 and 2014. Multivariate logistic regressions were used to identify predictors of ULMS CP and to evaluate the association between this complication and outcomes.
\end{abstract}

Results: During 10,373 ULMS-PCI procedures, CP occurred more frequently than in non-ULMS-PCI $(0.9$ vs. $0.4 \%, p<.001)$ with a stable annual incidence. Covariates associated with CP included number of stents used, female gender, use of rotational atherectomy and chronic total occlusion (CTO) intervention. Adjusted odds of adverse outcomes for ULMS-PCI complicated by CP were higher for peri-procedural complications including cardiogenic shock, tamponade, side-branch loss, DC cardioversion, in-hospital major bleeding, transfusion requirement, and peri-procedural myocardial infarction. There were also significantly increased odds for in-hospital major adverse cardiac events (MACCE, OR 8.961, 95\% Cl [4.902-16.383]) and 30-day mortality (OR 5.301, 95\% Cl [2.741-10.251]).

Conclusions: $\mathrm{CP}$ is an infrequent event during ULMS-PCI and is predicted by female gender, rotational atherectomy, CTO interventions or number of stents used. CP was associated with significantly higher odds of mortality and morbidity, but at rates similar to previously published all-comer $\mathrm{PCl}$ complicated by $\mathrm{CP}$.

KEYWORDS

complications, left main stem, outcomes, percutaneous coronary intervention, perforation 


\section{1 | INTRODUCTION}

Left main stem (LMS) coronary artery stenosis is associated with significant morbidity and mortality and has traditionally been treated by coronary artery bypass grafting (CABG). ${ }^{1}$ Recently, however, there has been an increasing amount of evidence emerging in favor of utilizing percutaneous coronary intervention $(\mathrm{PCl})$ in patients with unprotected LMS disease unprotected left main stem (ULMS-PCI). ${ }^{2}$ This has been the result of advancements in the $\mathrm{PCl}$ field, which saw the rise of using drug-eluting stents, the precision of intravascular imaging and the utility of risk stratification tools to refine patient selection. ${ }^{1}$

Coronary perforation (CP) is a rare but serious complication of $\mathrm{PCl}$ which has been shown to have an incidence of $\sim 0.4 \%$ of all procedures. ${ }^{3}$ The rate of perforations complicating ULMS interventions has been reported to be in the region of $1.2 \%$ from single-center experience. ${ }^{4}$ However, the literature surrounding ULMS perforation, its predictors and likely outcomes are very limited.

Studies examining predictors of perforation in all-comer $\mathrm{PCl}$ have suggested that increasing age, female gender, chronic total occlusion (CTO) intervention, number and length of stents used, and rotational atherectomy are associated with an increased rate with perforation. ${ }^{3,5}$ Overall, perforation was observed to lead to higher rates of 30-day mortality. ${ }^{3,5}$ Despite this, it is unclear whether these predictors and outcomes apply to ULMS-PCI complicated by perforation.

Therefore, the primary objective of this study was first to define the incidence, temporal trends, predictors, and outcomes of perforation associated with ULMS-PCI through analysis of the British Cardiovascular Society $(\mathrm{BCIS})$ national $\mathrm{PCI}$ database.

\section{2 | METHODS}

\subsection{Study design, setting, and participants}

We retrospectively analyzed national data from all patients undergoing ULMS-PCI in England and Wales between January 2007 and December 2014. During the study period, a total of 10,373 patients underwent ULMS-PCI. Patients were excluded if CP status was not recorded. The study was approved by review board of the National Institute of Clinical Outcomes Research and by the Healthcare Quality Improvement Partnership (HQIP).

\subsection{Setting, data source, and study size}

Data on $\mathrm{PCl}$ practice in the United Kingdom were obtained from the $\mathrm{BCIS}$ data set that records this information prospectively and publishes this information in the public domain as part of the national transparency agenda. ${ }^{6}$

The data collection process is overseen by The National Institute of Cardiovascular Outcomes Research (NICOR) (http://www.ucl.ac. $\mathrm{uk} /$ nicor/) with high levels of case ascertainment. The BCIS-NICOR database contains 121 clinical, procedural and outcomes variables, and in $2014,98.6 \%$ of all $\mathrm{PCl}$ procedures performed in the National Health Service (NHS) hospitals in England and Wales (www.bcis.org. $\mathrm{uk} /$ ) were recorded on the database with approximately 100,000 new records currently added each year. The accuracy and quality of the BCIS data set has previously been ascertained. ${ }^{7}$

Entry of all $\mathrm{PCl}$ procedures by $\mathrm{UK}$ interventional operators is mandated as part of professional revalidation. The participants of the database are tracked by the Medical Research Information Services for subsequent mortality using the patients' National Health Service (NHS) number (a unique identifier for any person registered within the NHS in England and Wales). Although the BCIS data set is UK wide, the participants of the database are tracked by linkage with life status information held by the Office of National Statistics (ONS) using each patient's unique NHS number, and therefore only patients from England and Wales have mortality data available.

\subsection{Study definitions}

We analyzed all recorded ULMS-PCI procedures that were undertaken in England and Wales between January 1, 2007 and December 31, 2014. CP was defined as in the BCIS guidance document as evidence of extravasation of dye or blood from any coronary artery during or following an interventional coronary procedure. Other study definitions were used as in the BCIS-NICOR database. Specifically, preprocedural renal failure is defined as any one of the following: creatinine $>200 \mu \mathrm{mol} / \mathrm{L}$, renal transplant history, or dialysis. Pre- or post-PCl disease severity was defined as a stenosis $\geq 50 \%$ in the case of the left main artery. Intravascular imaging was a combination of intravascular ultrasound and optical coherence tomography. An access site complication was defined as either a false aneurysm, hemorrhage (without hematoma), hemorrhage with delayed hospital-discharge, retroperitoneal hematoma, arterial dissection, or any access site complication requiring surgical repair. The clinical outcomes examined were inhospital mortality, in-hospital MACCE (defined as a combination death, peri-procedural stroke, or peri-procedural myocardial infarction after $\mathrm{PCl}$ ), in-hospital major bleeding (defined as either gastrointestinal bleed, intra-cerebral bleed, retroperitoneal hematoma, blood or platelet transfusion, access site hemorrhage, or an arterial access site complication requiring surgery), in-hospital reinfarction, in-hospital emergency cardiac surgery, tamponade, and 12-month mortality.

\section{4 | Data analyses}

The study flow is illustrated in Figure S1. Procedures with a protected LMS or missing LMS protection status were excluded as were procedures where the perforation status was blank. Statistical analysis was performed using the $\mathrm{R}$ coding environment (Open Source, version 3.5.1). Multiple imputations were carried out using the mice package to reduce the potential bias from missing data, assuming missing at random mechanisms. We used chained equations to impute the data for all variables with missing information and generated 10 data sets 
to be used in the analyses. We examined the baseline and procedural characteristics of participants by CP status. We explored crude baseline comorbidities using a Chi-squared test for categorical variables and the Wilcoxon-Mann-Whitney test for continuous variables.

A multiple logistic regression model was developed to identify variables associated with CP. The potential predictor variables in the model included age, body mass index (BMI), sex, smoking, hypertension, previous stroke, peripheral vascular disease, renal disease, previous $\mathrm{MI}$, $\mathrm{EF}<30 \%$, previous $\mathrm{PCl}$, diabetes, number of vessels diseased at baseline, CTO attempted, number of stents used, STEMI, Q wave on ECG, GPIIBIIIA inhibitor use, clopidogrel, prasugrel, ticagrelor, bivalirudin, radial access, dual access, largest balloon/stent, longest balloon/stent, vessels/ lesions attempted, in-stent restenosis attempted, thrombectomy, rotational atherectomy, imaging, operator status, penetration catheter, laser atherectomy, and micro-catheter use.

To examine the influence of perforation on ULMS-PCI outcomes, we built on and included the previously described baseline model to investigate the independent odds of shock, tamponade, cardioversion, dissection, side branch loss, slow flow phenomenon, gastrointestinal bleeding, transfusion, in-hospital major bleeding, peri-procedural myocardial infarction, arterial complication, acute kidney injury, in-hospital MACCE, in-hospital, 30 day, and 12 month death.

\section{3 | RESULTS}

\subsection{Incidence of CP and baseline demographics during ULMS-PCI by perforation status}

Crude numbers of ULMS-PCI increased significantly during the study period, as did ULMS-PCl activity of as a percentage of the total $\mathrm{PCl}$ (increasing from 2.0 to $4.1 \%, p<.001$ for trend, Figure $1 \mathrm{a} / \mathrm{b}$ ). During the study period, a total of 10,373 patients underwent ULMS-PCI for any indication, of whom 96 (0.9\%) experienced CP. The frequency of ULMS perforation was higher than in non-ULMS-PCI (0.9 vs. $0.4 \%$, $p<.001$ ). The total number of ULMS-PCI increased from 583 in 2007 to 2,030 in 2014 with a stable rate of perforation (Figure 1c). The baseline characteristics of ULMS-PCI patients with and without CP are presented in Table 1. CP was associated with increasing patient age, female sex, hypertension, and number of diseased vessels at baseline $(p<.05$, unadjusted).

\section{2 | Procedural variables during ULMS-PCI by perforation status}

The procedural variables for patients with and without $\mathrm{CP}$ by vessel type are presented in Table S1. ULMS-PCl cases with perforation were associated with more vessels and lesions attempted, CTO intervention, thrombus aspiration, rotational atherectomy, micro-catheter use, and the use of more stents during the procedure $(p<.05$, unadjusted)

\section{3 | Predictors of CP during ULMS-PCI in England and Wales 2007-2014}

Using multivariate analyses, only a limited number of covariates were found to be associated with CP during ULMS-PCI. After adjusting for baseline comorbidities, the only patient-related factor significantly associated with an increased incidence of perforation was female gender (odds ratio [OR] 1.887, 95\% confidence intervals [Cls] [1.134-3.141]). (a)

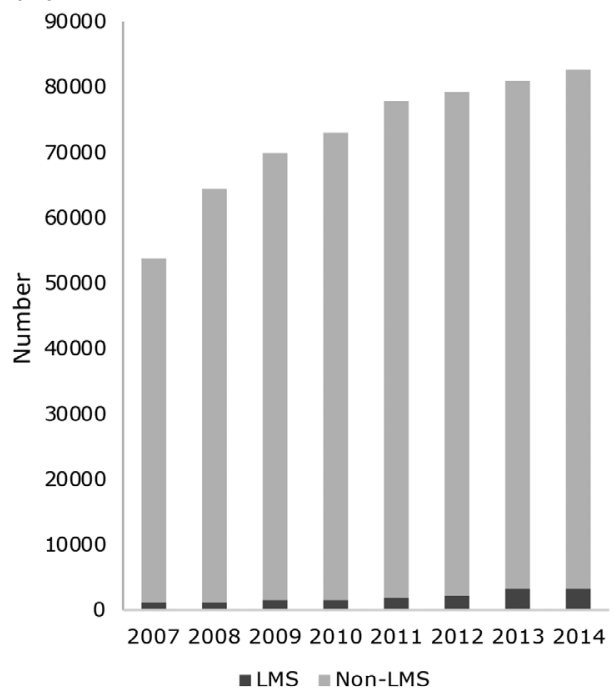

(b)

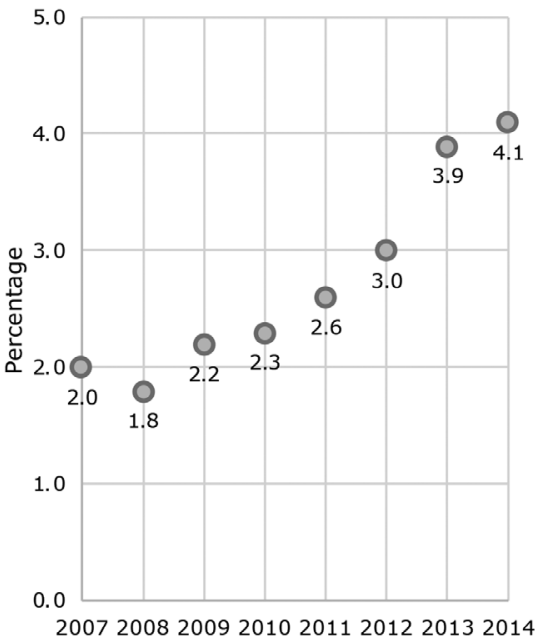

(c)

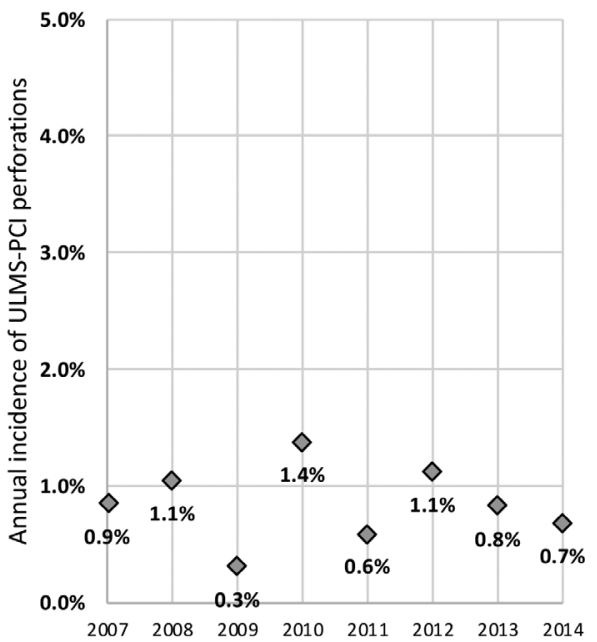

FIGURE 1 Trends in LMS-PCI performed in England and Wales 2007-2014. (a) Crude numbers of LMS (dark gray bars) and nonLMS-PCI (light gray bars); (b) percentage of total PCl performed represented by LMS-PCI ( $p<.001$ for trend); (c) percentage of ULMS-PCI complicated by perforations relative to all ULMS-PCI ( $p=.178$, not significant, for trend) 
TAB LE 1 Baseline participant characteristics by coronary perforation status in patients undergoing LMS-PCl in England and Wales 2007-2014

\begin{tabular}{|c|c|c|c|}
\hline Variables & Not perforated $(n=10,277)$ & Perforated $(n=96)$ & $p$ value \\
\hline Age (years), $\pm S D$ & $70.2 \pm 11.9$ & $73.8 \pm 10.4$ & .004 \\
\hline Female, no. (\%) & $2,952(28.8)$ & $38(39.6)$ & .027 \\
\hline Smoker, no. (\%) & $5,696(61.2)$ & $50(56.8)$ & .466 \\
\hline BMI $\left(\mathrm{kg} / \mathrm{m}^{2}\right), \pm S D$ & $28.2 \pm 12.8$ & $28 \pm 4.6$ & .480 \\
\hline Hypertension, no. (\%) & $6,141(62.8)$ & $65(73.9)$ & .043 \\
\hline Diabetes, no. (\%) & $2,418(24)$ & $23(25.6)$ & .826 \\
\hline Previous MI, no. (\%) & $3,718(37.6)$ & $36(39.6)$ & .783 \\
\hline Previous stroke, no. (\%) & $733(7.5)$ & $11(12.5)$ & .118 \\
\hline Peripheral vascular disease, no. (\%) & $1,080(11)$ & $13(14.8)$ & .340 \\
\hline Q wave on ECG, no. (\%) & $1,232(12.8)$ & $5(5.5)$ & .054 \\
\hline Renal disease, no. (\%) & $619(6.3)$ & $6(6.7)$ & 1.000 \\
\hline Creatinine ( $\pm \mathrm{mol} / \mathrm{L}), \pm S D$ & $107.8 \pm 75.4$ & $99.4 \pm 38.8$ & .859 \\
\hline Previous PCI, no. (\%) & $2,725(27)$ & $21(23.1)$ & .471 \\
\hline LVEF <30\%, no. (\%) & $813(12.6)$ & $8(10.7)$ & .744 \\
\hline Cardiogenic shock, no. (\%) & $0.0(0.0)$ & $0.0(0.0)$ & - \\
\hline ST elevation presentation, no. (\%) & $1,010(9.8)$ & $6(6.2)$ & .307 \\
\hline Clopidogrel & $7,637(89.2)$ & $74(85.1)$ & .288 \\
\hline Prasugrel & $314(3.7)$ & $2(2.3)$ & .686 \\
\hline Ticagrelor & $615(7.2)$ & $10(11.5)$ & .184 \\
\hline Warfarin, no. (\%) & $134(1.4)$ & $0.0(0.0)$ & - \\
\hline No. of vessels diseased at baseline, $\pm S D$ & $2.0 \pm 1.0$ & $2.3 \pm 1.0$ & .035 \\
\hline \multicolumn{4}{|l|}{ Number of procedures per year (\%) } \\
\hline 2007 & 578 (99.1\%) & $5(0.9 \%)$ & 1.000 \\
\hline 2008 & $656(98.9 \%)$ & $7(1.1 \%)$ & .884 \\
\hline 2009 & 948 (99.7\%) & $3(0.3 \%)$ & .057 \\
\hline 2010 & 994 (98.6\%) & $14(1.4 \%)$ & .152 \\
\hline 2011 & $1,326(99.4 \%)$ & $8(0.6 \%)$ & .239 \\
\hline 2012 & $1,578(98.9 \%)$ & $18(1.1 \%)$ & .446 \\
\hline 2013 & 1,667 (99.2\%) & $14(0.8 \%)$ & .773 \\
\hline 2014 & 2016 (99.3\%) & $14(0.7 \%)$ & .270 \\
\hline
\end{tabular}

Abbreviations: BMI, body mass index; LMS, left main stem; MI, myocardial infarction; PCI, percutaneous coronary infection; SD, standard deviation.

Procedural variables significantly associated with an adjusted increased risk of perforation were number of stents used $(O R 1.390,95 \% \mathrm{Cl}$ [1.166-1.656]), number of CTO attempted (OR 2.036, 95\% C [1.350-3.072]) and use of rotational atherectomy (OR 2.494, 95\% Cl [1.305-4.765]). The full model with all covariates can be seen in Table S2.

\section{4 | Clinical outcomes of ULMS-PCI by perforation status}

The unadjusted incidence of procedural complications associated with CP in ULMS-PCl is shown in Table S3. Complications crudely associated with $\mathrm{CP}$ were shock, tamponade, cardioversion, major side branch loss, more residual disease, transfusion, in-hospital major bleeding, peri-procedural $\mathrm{MI}$, and in-hospital MACCE $(p<.05)$. Unadjusted mortality rates at 30 days, at 12 months and in-hospital were higher in cases where perforation occurred.

Multivariate logistic modeling was used to adjust outcomes for baseline comorbidities (Table 2). This showed that perforation complicating ULMS-PCI inferred significantly higher odds of in-hospital major adverse cardiac events (MACCE, OR 8.961, 95\% Cl [4.902-16.383]), 30-day mortality (OR 5.301, 95\% Cl [2.741-10.251]), and 12-month mortality (OR 2.412, 95\% Cl [1.325-4.390]). There were also higher odds of peri-procedural complications, such as shock, tamponade, DCCV, side-branch loss, inhospital major bleed, transfusion requirement, and peri-procedural MI $(p<.05)$. 
TABLE 2 Outcomes by coronary perforation status in patients undergoing LMS-PCl in England and Wales 2007-2014
FIGURE 2 Outcomes of coronary perforation in patients undergoing unprotected LMS-PCl and all $\mathrm{PCl}^{3}$ Odds ratio with $95 \%$ confidence intervals demonstrating that outcomes of perforation in ULMS-PCl are no worse than all-comer $\mathrm{PCl}$

\begin{tabular}{|lcccc|}
\hline Variable & Odds ratio & Lower Cl & Upper Cl & $p$ value \\
\hline Shock induced by procedure & 14.884 & 6.540 & 33.873 & $<.001$ \\
\hline DCCV & 4.427 & 1.136 & 17.247 & .032 \\
\hline Dissection & 1.092 & 0.487 & 2.448 & .830 \\
\hline Side-branch loss & 13.172 & 5.846 & 29.677 & $<.001$ \\
\hline Slow flow & 1.781 & 0.387 & 8.202 & .459 \\
\hline Arterial complication & 1.211 & 0.247 & 5.944 & .813 \\
\hline Transfusion & 8.648 & 2.852 & 26.227 & $<.001$ \\
\hline GI bleed & 7.992 & 0.921 & 69.365 & .059 \\
\hline In-hospital major bleed & 30.019 & 15.158 & 59.450 & $<.001$ \\
\hline Peri-procedural MI & 9.019 & 3.380 & 19.025 & $<.001$ \\
\hline Acute kidney injury & 2.190 & 0.266 & 18.029 & .466 \\
\hline In-hospital death & 8.633 & 4.097 & 18.192 & $<.001$ \\
\hline In-hospital MACCE & 8.961 & 4.902 & 16.383 & $<.001$ \\
\hline 30-day mortality & 5.301 & 2.741 & 10.251 & $<.001$ \\
\hline 12-month mortality & 2.412 & 1.325 & 4.390 & .004 \\
\hline
\end{tabular}

Abbreviations: $\mathrm{Cl}$, confidence interval; LMS, left main stem; MACCE: major adverse cardiac and cerebrovascular events; $\mathrm{MI}$, myocardial infarction; $\mathrm{PCl}$, percutaneous coronary infection.

\section{2 month mortality}

\section{0 day mortality}

\section{In-hospital MACCE}

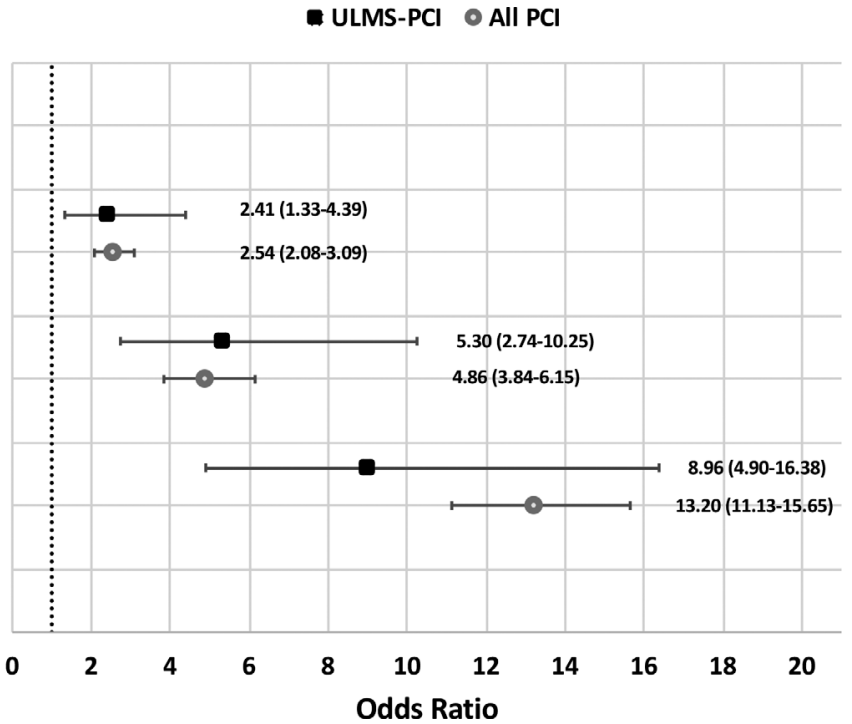

Compared to reports on perforation complicating all-comer $\mathrm{PCl}$, the rates of perforation in ULMS-PCl were higher $(0.9 \%$ vs. the reported $0.4 \%)^{3}$ This likely reflects the complex/high risk nature of patients requiring this procedure and is consistent with other studies of patients with complex coronary anatomy, such as those undergoing $\mathrm{PCl}$ with CTO disease where higher rates of perforation were observed (1.4\%). ${ }^{8}$ Indeed, we found that CTO is a complicating factor and a predictor of perforation in ULMS-PCI, consistent with previous studies. ${ }^{8}$ Despite the increase in the overall number of ULMS-PCI over the study period, there was no significant change in the rate of perforation. The observational nature of the study makes it difficult to determine the reasons for this, but we suspect that improvement in 
toolkit safety profile over the study period may have offset any noticeable increase in the incidence of perforation.

In-hospital MACCE and mortality ( 30 days and 12 months) were significantly more likely in ULMS-PCl complicated by perforation, inferring a fivefold increased odds of death at 30 days (Table 2). These data are a stark reminder that although perforation during ULMS-PCI is a relatively rare event, when it does occur, there is an important association with poor outcome. However, compared to previously published studies on all-comer $\mathrm{PCl}$ complicated by perforation, ${ }^{3}$ the odds ratios of in-hospital MACCE and mortality are comparable (Figure 2). This suggests that although the rates of perforation in ULMS-PCI are just over twice that in all-comer $\mathrm{PCl}$, the outcomes are no worse and therefore should not detract from carrying out ULMS PCI when indicated.

The limited number of predictors associated with perforation shown in Table S2 makes it difficult to anticipate its occurrence. Indeed, in our study, with the exception of rotational atherectomy, the occurrence of perforation does not seem to be significantly associated with procedural variables (e.g., use of microcatheters, choice of access, or antiplatelet agent). The association we observed with the number of stents used may reflect the perforation treatment strategy deployed by the operator. Consequently, operators need to recognize this limitation of prediction and be prepared to tackle such complication arising by having the necessary tools, algorithms, and expertise on board in order to reduce the rate of the adverse outcomes described above (Table 2).

The complications that we have reported to be significantly associated with perforation in ULMS-PCI are likely related to the occurrence of perforation (shock, tamponade, in-hospital major bleed) and/or as a consequence of its treatment (shock, side-branch loss, peri-procedural MI, DCCV, transfusion) and are consistent with previous reports. ${ }^{9,10}$ Indeed, the relatively recent advances in the treatment of $\mathrm{CP}$ with covered stents are likely responsible for the higher rates we have observed with major side branch loss and periprocedural shock seen in our study, both of which shown to be strongly predictive of mortality., ${ }^{9,11}$

This analysis has several strengths. The BCIS data set includes >98\% of all $\mathrm{PCl}$ procedures performed in the United Kingdom, which, therefore, reflects a national, real-world experience that includes high-risk patients encountered in daily interventional practice (who are often excluded from randomized controlled trials). Such large national registry data with unselected enrolment are important for evaluation of low-frequency complications, such as CP, particularly given that such low event rates would mean that single-center registries or randomized controlled trials would be grossly underpowered.

\section{1 | Limitations}

The BCIS database does not differentiate between CPs resulting from guide-wire and those perforations due to balloon or stent inflation or indeed the anatomical location of such perforations. Second, the database does not record guidewire data and therefore data on stiffness or coating and the incidence of CP cannot be provided. Third, the database does not record the Ellis classification of CP so that a substratification by perforation severity was not possible in this series. Moreover, the $\mathrm{BCIS}$ database does not record the use of other treatment strategies such covered stents, pericardial drains, or embolization techniques and therefore data on outcomes with respect to different therapies is not available. Furthermore, the follow-up period is limited to 2014 as more recent data with reliable linkage to outcomes are not available to the authors. Finally, because of the observational nature of this study, any conclusions may be influenced by unmeasured confounders, such as frailty or anatomical considerations.

\section{5 | CONCLUSIONS}

$\mathrm{CP}$ is an infrequent event during ULMS-PCl with very few predictors and a steady incidence over the study period of 7 years. It is associated with significantly adverse peri-procedural outcomes as well as increased morbidity and mortality. However, the likelihood of occurrence of these outcomes is similar to previous studies that examined perforation in all-comer $\mathrm{PCl}$ and therefore should not be a basis for avoiding indicated ULMS-PCI.

\section{CONFLICT OF INTEREST}

The authors declare no potential conflict of interest.

\section{ORCID}

Majd B. Protty (D) https://orcid.org/0000-0001-8992-9120

Richard Anderson (D) https://orcid.org/0000-0003-0582-1628

Tim Kinnaird (D) https://orcid.org/0000-0003-1209-3534

\section{REFERENCES}

1. Rampat R, Hildick-Smith D. Left main stem percutaneous coronary intervention - data and ongoing trials. Interv Cardiol. 2015;10: 132-135.

2. Kindi HA, Samaan A, Hosny H. NOBLE and EXCEL: the debate for excellence in dealing with left main stenosis. Glob Cardiol Sci Pract. 2018;2018:3

3. Kinnaird T, Kwok CS, Kontopantelis E, et al. Incidence, determinants, and outcomes of coronary perforation during percutaneous coronary intervention in the United Kingdom between 2006 and 2013: an analysis of 527121 cases from the British cardiovascular intervention society database. Circ Cardiovasc Interv. 2016;9(8):e003449. https:// doi.org/10.1161/CIRCINTERVENTIONS.115.003449.

4. Alaour B, Onwordi E, Khan A, Dana A. Clinical outcome of left Main stem (LMS) percutaneous coronary intervention $(\mathrm{PCI})$ in a large nonsurgical UKCenter: a 5-year clinical experience. J Am Coll Cardiol Intv. 2018;11(4):S16-S17.

5. Kinnaird T, Calvert P, Anderson R, et al. Coronary perforation complicating percutaneous coronary intervention in patients presenting with an acute coronary syndrome: an analysis of 1,013 perforation cases from the British cardiovascular intervention society database. J Am Coll Cardiol. 2018;72(299):37-42.

6. British Cardiovascular Intervention Society. BCIS Audit Returns for Adult Interventional Procedures Jan 2015-Dec 2015.

7. Ludman PF, British Cardiovascular Intervention S. British cardiovascular intervention society registry for audit and quality assessment of percutaneous coronary interventions in the United Kingdom. Heart. 2011;97:1293-1297. 
8. Kinnaird T, Anderson R, Ossei-Gerning N, et al. Legacy effect of coronary perforation complicating percutaneous coronary intervention for chronic total occlusive disease: an analysis of 26807 cases from the British cardiovascular intervention society database. Circ Cardiovasc Interv. 2017;10(5): e004642. https://doi.org/10.1161/CIRCINTERVENTIONS.116.004642.

9. Nguyen-Trong PK, Rangan BV, Karatasakis A, et al. Predictors and outcomes of side-branch occlusion in coronary chronic total occlusion interventions. J Invasive Cardiol. 2016;28:168-173.

10. Gorenek B, Lundqvist CB, Terradellas JB, et al. Cardiac arrhythmias in acute coronary syndromes: position paper from the joint EHRA, ACCA, and EAPCI task force. Eur Heart J Acute Cardiovasc Care. 2015;4:386.

11. Wisniewska-Szmyt J, Kubica J, Sukiennik A, et al. One-year outcomes of left main coronary artery stenting in patients with cardiogenic shock. Cardiol J. 2007;14:67-75.

\section{SUPPORTING INFORMATION}

Additional supporting information may be found online in the Supporting Information section at the end of this article.

How to cite this article: Hussain HI, Protty MB, Gallagher S, et al. The impact of coronary perforation in percutaneous interventions involving the left main stem coronary artery in the United Kingdom 2007-2014: Insights from the British Cardiovascular Intervention Society database. Catheter Cardiovasc Interv. 2020;1-7. https://doi.org/10.1002/ccd. 28933 